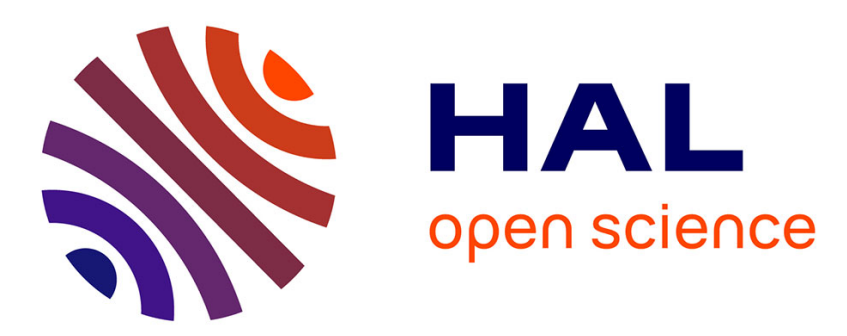

\title{
Trajectory Free Linear Model Predictive Control for Stable Walking in the Presence of Strong Perturbations
}

\author{
Pierre-Brice Wieber
}

\section{To cite this version:}

Pierre-Brice Wieber. Trajectory Free Linear Model Predictive Control for Stable Walking in the Presence of Strong Perturbations. IEEE-RAS International Conference on Humanoid Robots, 2006, Genova, Italy. inria-00390462

\section{HAL Id: inria-00390462 https://hal.inria.fr/inria-00390462}

Submitted on 2 Jun 2009

HAL is a multi-disciplinary open access archive for the deposit and dissemination of scientific research documents, whether they are published or not. The documents may come from teaching and research institutions in France or abroad, or from public or private research centers.
L'archive ouverte pluridisciplinaire HAL, est destinée au dépôt et à la diffusion de documents scientifiques de niveau recherche, publiés ou non, émanant des établissements d'enseignement et de recherche français ou étrangers, des laboratoires publics ou privés. 


\title{
Trajectory Free Linear Model Predictive Control for Stable Walking in the Presence of Strong Perturbations
}

\author{
Pierre-Brice Wieber \\ INRIA Rhône-Alpes \\ 38331 St Ismier Cedex, France \\ Email: Pierre-Brice.Wieber@inrialpes.fr
}

\begin{abstract}
A humanoid walking robot is a highly nonlinear dynamical system that relies strongly on contact forces between its feet and the ground in order to realize stable motions, but these contact forces are unfortunately severely limited. Model Predictive Control, also known as Receding Horizon Control, is a general control scheme specifically designed to deal with such contrained dynamical systems, with the potential ability to react efficiently to a wide range of situations. Apart from the question of computation time which needs to be taken care of carefully (these schemes can be highly computation intensive), the initial question of which optimal control problems should be considered to be solved online in order to lead to the desired walking movements is still unanswered. A key idea for answering to this problem can be found in the ZMP Preview Control scheme. After presenting here this scheme with a point of view slightly different from the original one, we focus on the problem of compensating strong perturbations of the dynamics of the robot and propose a new Linear Model Predictive Control scheme which is an improvement of the original ZMP Preview Control scheme.
\end{abstract}

\section{INTRODUCTION}

A humanoid walking robot is a highly nonlinear dynamical system that relies strongly on contact forces between its feet and the ground in order to realize stable motions, but these contact forces are unfortunately severely limited [1]. Classical trajectory tracking control laws are structurally unable to deal with such strong constraints on the dynamics of a system, especially when having to face strong perturbations. They have been regularly completed therefore with higher-level adaptation schemes of the trajectories being tracked [2], [3], [4], [5], [6]. But all of these schemes are based on predefined sets of possible motions which may not be able to adapt to all the situations a humanoid robot may have to face. There is a need therefore for a scheme that would generate online the motions that a humanoid robot needs to realize by continuously taking care of its dynamical state.

Model Predictive Control, also known as Receding Horizon Control, is a general control scheme specifically designed to deal with such contrained dynamical systems and generate online the motions that need to be realized, with therefore the potential ability to react efficiently to a wider range of situations [7], [8], [9]. It globally amounts to solving online a sequence of Optimal Control problems. Such a scheme has been already applied successfully to biped walking robots [10], but apart from the question of computation time which needs to be taken care of carefully (these schemes can be highly computation intensive), the question of which optimal control problems should be considered to be solved online in order to lead to the desired walking movements is still unanswered. This question is of particular interest because of the limited horizon over which computations can be carried out: the problem is to find which optimal control problem can lead to stable long term motions while being solved only over short horizons.

A key idea for answering to this problem can be found in the ZMP Preview Control scheme proposed in [11] in order to generate dynamically stable motions through a 3D Linear Inverted Pendulum approximation of the dynamics of the Center of Mass of a humanoid robot [11], [12], [13]. We propose here to analyze how strong perturbations of a humanoid robot can be dealt with efficiently with such a scheme and how it can be improved.

After presenting the ZMP Preview Control scheme in section II with a point of view slightly different from the original presentation given in section III, a brief stability analysis is proposed in section III before focusing on the problem of compensating strong perturbations of the dynamics of the robot in section IV and proposing a new Linear Model Predictive Control scheme which is an improvement of the original ZMP Preview Control scheme in section V.

\section{THE ZMP PREVIEW CONTROL SCHEME REVISITED}

The most severe restriction on the realization of stable motions by humanoid robots is that they require contact forces with the environment which are generally very strictly limited by the physics of contacting bodies. When walking on a flat ground, this boils down to the fact that the Center of Pressure (CoP), also called the Zero Moment Point (ZMP) can lie only within the convex hull of the contact points between the robot's feet and the ground (more complex situations, involving contacts of the hands or even simply walking upstairs oblige working directly with the wrench of the contact forces [1]).

A general approximation to the position of the CoP is to neglect the inertial effects due to rotations of the different parts of the robot. If we suppose moreover that the Center of Mass (CoM) of the robot doesn't move vertically, we end up 
with a position $z$ of the CoP on the ground being simply:

$$
z=x-\frac{h_{C o M}}{g} \ddot{x}
$$

where $x$ is the horizontal position of the CoM, $\ddot{x}$ its horizontal acceleration, $h_{C o M}$ its altitude and $g$ the norm of the gravity force. This approximation naturally decouples the forward and lateral motions of the robot in the analysis of its CoP. We will focus therefore throughout this article on lateral motions only, knowing that the case of forward motions is absolutely identical.

Introduced for the first time in the reference [11], the ZMP Preview Control scheme proposes to generate a trajectory of the CoM of a humanoid robot under the constraint that the footsteps are fixed and impossible to change. The constraint is therefore that the trajectory of the CoP given by equation (1) always stays within the convex hull of these fixed footprints. An additionnal simplifying assumption is that the altitude $h_{C o M}$ of the CoM be constant.

The trajectories of both the CoM and the CoP are discretized then as piecewise cubic polynomials, with constant jerks $\dddot{x}$ and $\dddot{z}$ over time intervals of constant lengths $T$. Focusing on the state of the system at times $t=k T$ with $k=1,2, \ldots$ with the notations

$$
\hat{x}_{k}=\left[\begin{array}{c}
x(k T) \\
\dot{x}(k T) \\
\ddot{x}(k T)
\end{array}\right], \dddot{x}_{k}=\dddot{x}(k T), z_{k}=z(k T),
$$

the trivial integration of the constant jerk $\dddot{x}_{k}$ over the time intervals of lengths $T$ leads to the recursive relationship

$$
\hat{x}_{k+1}=\left[\begin{array}{ccc}
1 & T & T^{2} / 2 \\
0 & 1 & T \\
0 & 0 & 1
\end{array}\right] \hat{x}_{k}+\left[\begin{array}{c}
T^{3} / 6 \\
T^{2} / 2 \\
T
\end{array}\right] \dddot{x}_{k}
$$

while the equation (1) leads to

$$
z_{k}=\left[\begin{array}{lll}
1 & 0 & h_{C o M} / g
\end{array}\right] \hat{x}_{k} .
$$

This way, the constraint on the position of the $\mathrm{CoP}$ appears to be simply

$$
z_{k}^{\min } \leq z_{k} \leq z_{k}^{\max }
$$

where the minimal and maximal admissible values depend on the horizontal position of the feet on the ground at each time $k T$.

This way, designing a trajectory of the CoM amounts to deciding a series of jerks $\dddot{x}_{k}$ such that after application of the recursive relationship (3)-(4), the constraints (5) are satisfied. We can observe now that for this linear recursive relationship under constraints, an unstable motion of the CoM is directly a motion diverging to $\pm \infty$ for which the jerk would also diverge to $\pm \infty$. The idea behind the ZMP Preview Control scheme proposed in the reference [11] is therefore to minimize this jerk while maintaining a position $z_{k}$ of the $\mathrm{CoP}$ as close as possible to some prescribed reference positions $z_{k}^{\text {ref }}$. This reference can be taken for example in the middle of the admissible values

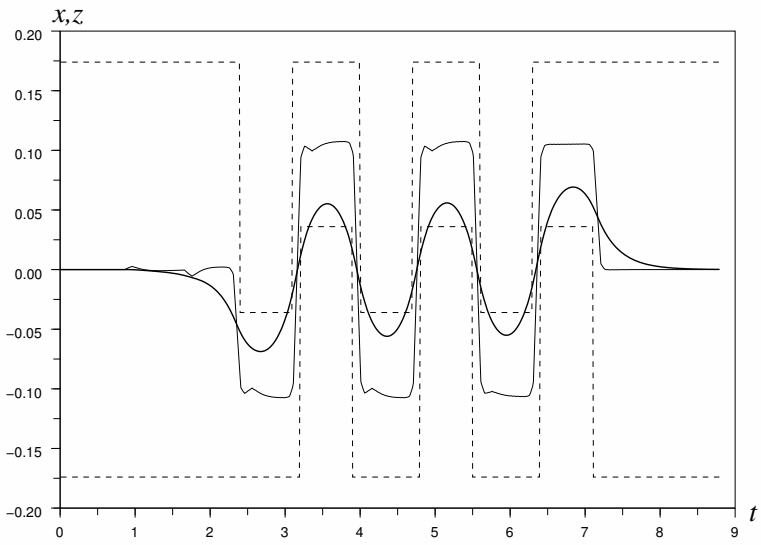

Fig. 1. Evolution with time (in seconds) of the lateral position of the CoM (thick curve) of a humanoid robot making 6 steps, together with the position of the CoP approximated by equation (1) (thin curve). The dashed curves represent the minimal and maximal admissible values for the $\mathrm{CoP}$, and we can observe that the CoP always stays inside this set of admissible values.

introduced in the constraints (5). This corresponds to solving at time $k T$ the Quadratic Program

$$
\dddot{x}_{k}, \min _{\dddot{x}_{k+1}, \ldots} \sum_{i=k}^{\infty} \frac{1}{2} Q\left(z_{i+1}-z_{i+1}^{r e f}\right)^{2}+\frac{1}{2} R \dddot{x}_{i}^{2}
$$

where the ratio $R / Q$ allows to balance the minimization of the jerks $\dddot{x}_{i}$ with the tracking of the reference positions $z_{i}^{r e f}$.

The idea of Receding Horizon Control, or Model Predictive Control [7], [8], [9] is to execute only the first interval $[k T,(k+1) T]$ of this trajectory, then measure the actual state of the system, here the position and velocity of the CoM, and then recompute a new trajectory with the same QP (6) but taking care of this new measure, allowing therefore for some feedback of the state. A last observation is that solving a simplified version of this original QP over a finite time interval $[k T,(k+N) T]$ is sufficient:

$$
\min _{\dddot{x}_{k}, \ldots \dddot{x}_{k+N}} \sum_{i=k}^{k+N-1} \frac{1}{2} Q\left(z_{i+1}-z_{i+1}^{r e f}\right)^{2}+\frac{1}{2} R \dddot{x}_{i}^{2} .
$$

Figure 1 shows a typical result of this whole procedure with $g=9.81 \mathrm{~m} . \mathrm{s}^{-2}, h_{C o M}=0.8 \mathrm{~m}$, a ratio $R / Q=10^{-6}$, $T=5 \mathrm{~ms}$ and $N=300$, inducing a pre-computation of trajectories over intervals of $1.5 \mathrm{~s}$ brought up to date every $5 \mathrm{~ms}$. This figure shows the evolution with time of the lateral position of the CoM (thick curve) of a humanoid robot making 6 steps, together with the position of the CoP approximated by equation (1) (thin curve). The dashed curves represent the minimal and maximal admissible values for the CoP, which depend on the different contact phases, here 6 single support phases, 3 on each foot, and double support phases between each single support phases and at the beginning and at the end of the whole trajectory.

Figure 2 shows the same results but with the evolution of the position of the $\mathrm{CoP}$ evaluated this time with the whole 


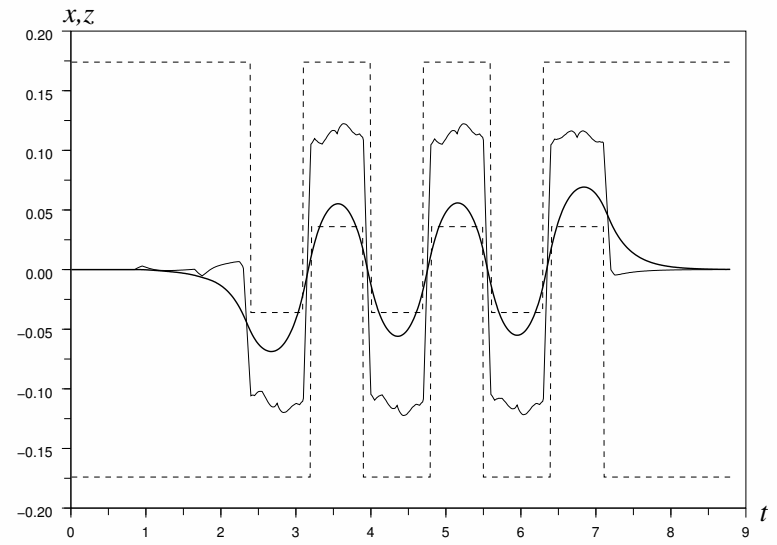

Fig. 2. Same results as in figure 1, but with the position of the CoP (thin curve) evaluated this time with the whole dynamical model of the robot.

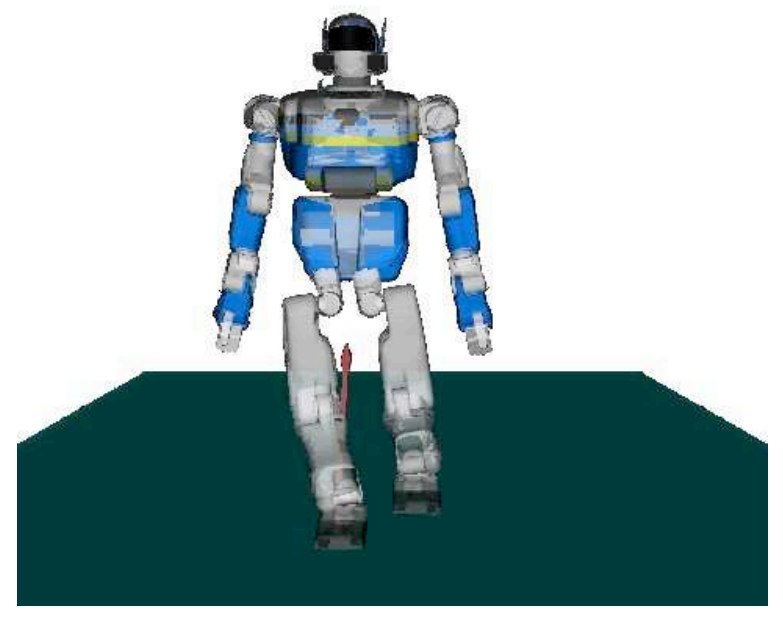

Fig. 3. Dynamical simulation of the HRP-2 robot.

dynamical model of the robot, here a HRP-2 robot (figure 3). For this whole dynamical model, the precise movements of the limbs have been simply defined by hand with splines in the cartesian space. We can observe in these two figures that the difference between the position of the CoP evaluated with the whole dynamical model and with the simple approximation (1) is always less than $2 \mathrm{~cm}$, both of these positions always staying well inside the set of admissible values, as desired. This scheme generates trajectories of the CoM that can be realized properly by a humanoid robot in most cases away from perturbations, and it is effectively used day after day for experiments on the HRP-2 robot [14], [15].

\section{Analytical Solution And Stability Analysis}

Considering the Quadratic Program (7) over a finite time interval instead of the original QP (6) over an infinite time in- terval allows solving the Optimal Control problem analytically through some simple matrix manipulations instead of having to solve a more complex algebraic Riccati equation [15]. A straightforward stability analysis will help us then in concluding that these matrix manipulations can indeed be made very fast.

The recursive relation (3) can be iterated $N$ times and combined with $N$ versions of the relation (4) in order to relate at once $N$ values of the jerk $\dddot{x}_{k}$ of the CoM with $N$ values of the position $z_{k}$ of the CoP:

$$
\begin{aligned}
& {\left[\begin{array}{c}
z_{k+1} \\
\vdots \\
z_{k+N}
\end{array}\right]=\left[\begin{array}{ccc}
1 & T & T^{2} / 2-h_{C o M} / g \\
\vdots & \vdots & \vdots \\
1 & N T & N^{2} T^{2} / 2-h_{C o M} / g
\end{array}\right] \hat{x}_{k}+} \\
& {\left[\begin{array}{ccc}
T^{3} / 6-T h_{C o M} / g & 0 & 0 \\
\vdots & \ddots & 0 \\
\left(1+3 N+3 N^{2}\right) T^{3} / 6-T h_{C o M} / g & \ldots & T^{3} / 6-T h_{C o M} / g
\end{array}\right]} \\
& \times\left[\begin{array}{c}
\dddot{x}_{k} \\
\vdots \\
\dddot{x}_{k+N-1}
\end{array}\right]
\end{aligned}
$$

where the second big matrix is a $N \times N$ lower triangular Toeplitz matrix, i.e. with constant diagonals. This relation can be considered in the more compact presentation

$$
Z_{k+1}=P_{x} \hat{x}_{k}+P_{u} \dddot{X}_{k}
$$

with which the Quadratic Program (7) can be simply rewritten as:

$$
\min _{\dddot{X}_{k}} \frac{1}{2} Q\left(Z_{k+1}-Z_{k+1}^{r e f}\right)^{2}+\frac{1}{2} R \dddot{X}_{k}^{2}
$$

This QP can be solved analytically then, leading to

$$
\dddot{X}_{k}=-\left(P_{u}^{T} P_{u}+\frac{R}{Q} I_{N \times N}\right)^{-1} P_{u}^{T}\left(P_{x} \hat{x}_{k}-Z_{k}^{r e f}\right)
$$

where $I_{N \times N}$ is an identity matrix. This way, the "control" applied to the dynamics (3)-(4) appears to be simply

$$
\dddot{x}_{k}=e^{T} \dddot{X}_{k},
$$

with $e=[1,0 \ldots 0]^{T}$. Considering a more compact presentation of the recursive relation (3),

$$
\hat{x}_{k+1}=A \hat{x}_{k}+B \dddot{x}_{k},
$$

verifying the stability of this whole control scheme amounts to verifying that the norms of the 3 eigenvalues of the matrix

$$
A-B e^{T}\left(P_{u}^{T} P_{u}+\frac{R}{Q} I_{N \times N}\right)^{-1} P_{u}^{T} P_{x}
$$

are smaller than 1.

Not surprisingly, we can observe by verifying this last point numerically that the stability of this control scheme depends on both the value of the ratio $R / Q$ and the length $N T$ of the horizon over which the trajectories are pre-computed, with reasonnable values being $R / Q=10^{-6}$ and $N T=1.5 \mathrm{~s}$. Interestingly enough, the number $N$ of pieces of this trajectory has nearly no influence on this stability so it can be kept low 


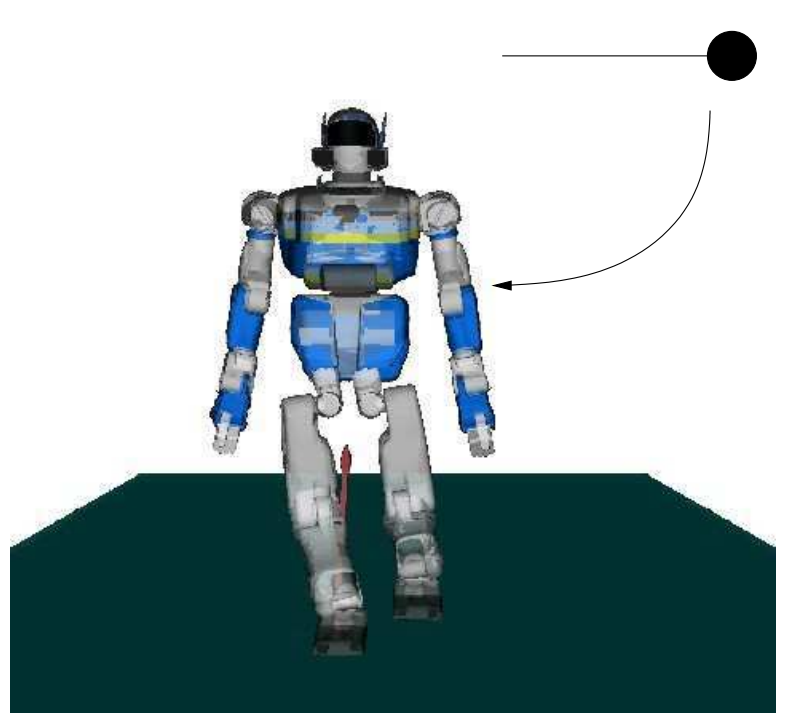

Fig. 4. The perturbation considered here corresponds to a mass $M$ hitting the robot in the middle of the trunk from the side after having fallen from a height of $0.5 \mathrm{~m}$ under the action of gravity.

as long as the final length $N T$ is kept constant. Keeping this number of pieces low allows dealing with small matrices, $20 \times 20$ or $30 \times 30$ when solving the QP (7) with the equation (11), what leads to extremely fast computations on modern computers.

\section{Dealing with Strong Perturbations}

Let's consider now the robot executing the trajectory designed in section II and having to deal with a strong perturbation. Let's consider for example that a mass $M$ hits the robot in the middle of the trunk from the side after having fallen from a height of $0.5 \mathrm{~m}$ under the action of gravity as described in figure 4, and let's consider that this happens at time $t=2.5 \mathrm{~s}$, in the beginning of the first single support phase.

Classical tracking control laws such as a computed torque with precompensation of contact forces (as appearing in [16]) can be used to track this predefined trajectory and absorb some perturbations. Figure 5 shows the result of a simulation of such a situation with a mass that hits the robot corresponding to $4 \%$ of the total mass of the robot (a bit more than $2 \mathrm{~kg}$ ). We can observe that the perturbation at time $t=2.5 \mathrm{~s}$ is correctly compensated and the robot manages to realize the 6 steps without any major failure, except for some error in the tracking of the feet positions on the ground, and some disturbances at impact times. Unfortunately, stronger perturbations can't be compensated anymore this way and they lead to a fall. Indeed, we can observe in figure 5 that the $\mathrm{CoP}$ reaches the limit of admissible values quickly after the occurrence of the perturbation, indicating that we're reaching the limits of this classical way of compensating perturbations.

Instead of solely relying on a classical tracking control law for compensating perturbations, we can make use of the feedback possibilities of the Model Predictive Control law

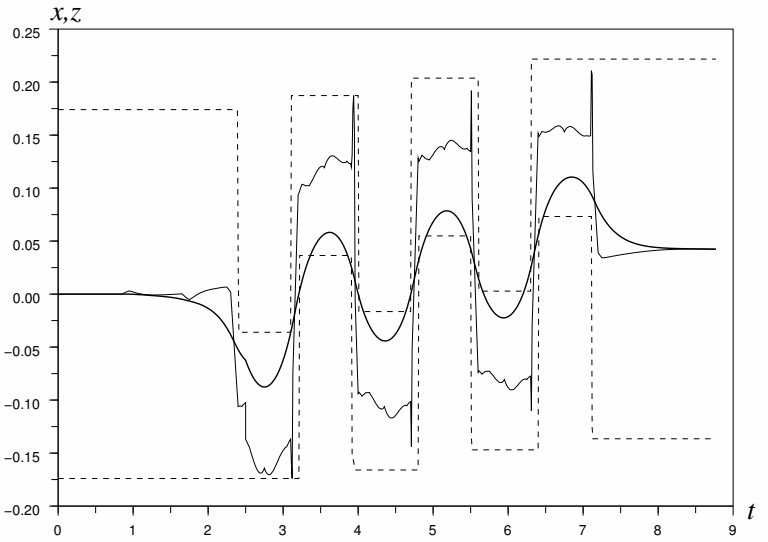

Fig. 5. Simulation of tracking the trajectory of figure 2 with a computed torque control law with precompensation of contact forces when a mass corresponding to $4 \%$ of the total mass of the robot hits the robot at time $t=2.5 \mathrm{~s}$

presented in section II and recompute online the trajectory of the CoM of the robot while continuously taking into account its actual state. Figure 6 shows the result of this online recomputation when the mass that hits the robot corresponds to $20 \%$ of the total mass of the robot (a bit more than $11 \mathrm{~kg}$ ). We can see that even though 5 times stronger than the perturbation considered previously, this perturbation is perfectly compensated here. In order to ensure that this trajectory can be realized safely, it is wiser from now on to consider limits on the position of the CoP $4 \mathrm{~cm}$ inside the feet. These are the limits that appear on figure 6 , and we can see that they are always satisfied. But we can observe also that they are reached once again quickly after the occurrence of the perturbation: stronger perturbations might not be compensated safely anymore with this scheme.

\section{EXPlicitely TAKING INTO ACCOUNT THE LiMITS ON THE POSITION OF THE COP}

The problem with the ZMP Preview Control scheme presented in section II is that the limits on the position of the $\mathrm{CoP}$ are not taken into account explicitely. We can reconsider therefore the idea of minimizing the jerk of the trajectory of the CoM, but balancing this minimization with maintaining explicitely the position of the $\mathrm{CoP}$ within the admissible values, leading to the following Quadratic Program with inequality constraints:

$$
\begin{gathered}
\min _{\dddot{X}_{k}} \frac{1}{2} \dddot{X}_{k}^{2} \\
Z_{k}^{\text {min }} \leq Z_{k} \leq Z_{k}^{\max }
\end{gathered}
$$

Of course, the solution to such a Quadratic Program with inequality constraints can be more costly to compute than the analytical solution (11) of the original one without constraints. But remembering the remark of the end of section III on the possibility to use only small matrices, such a small QP can 


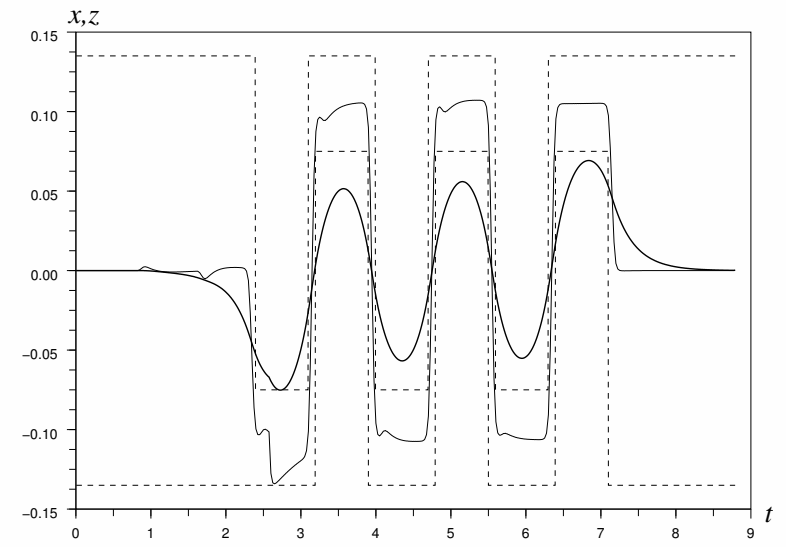

Fig. 6. Online recomputation of the trajectory of the Center of Mass of the robot with the ZMP Preview Control scheme, taking into account the perturbation at time $t=2.5 \mathrm{~s}$ generated by a mass corresponding to $20 \%$ of the total mass of the robot. Note that the limits on the position of the CoP that appear here and in the following figures lie $4 \mathrm{~cm}$ inside the feet for safety reasons.

be solved in fact in a fraction of a milli-second on modern computers.

Figure 7 shows the same results as figure 1, but using this new QP instead of the one of section II, and we can verify that it is effective in generating a stable trajectory of the CoM. Figure 8 shows how this new QP allows to compensate a perturbation generated by a mass hitting the trunk of the robot that corresponds to $33 \%$ of the total mass of the robot (a bit more than $18 \mathrm{~kg}$ ), more than 1.5 times the previous perturbation. We can observe in this figure how the $\mathrm{CoP}$ is always kept strictly inside the admissible region, here $4 \mathrm{~cm}$ inside the true admissible region for safety reasons, while slowly compensating the perturbation in 2 steps. We can finally verify in figure 9 that the evolution of the position of the CoP evaluated with the whole dynamical model of the robot always stays well inside the true set of admissible values, ensuring that this trajectory can indeed be realized safely

Note that the stability analysis of general Model Predictive Control schemes such as the one proposed here is possible [8], but can be far more complex than the analysis of the norms of eigenvalues realized in section III since we can't rely here on any analytical solution of the underlying optimization problem such as the equation (11).

\section{CONCLUSION}

The online adaptation of the choice of the reference trajectory proposed in [6] for the control of a $40 \mathrm{~kg}$ walking robot allowed to compensate a perturbation of $750 \mathrm{~N}$ in the sagittal plane during $25 \mathrm{~ms}$. This would be analogous to a mass $M$ corresponding to $15 \%$ of the total mass of the robot hitting it as in figure 4: the scheme proposed here appears to perform much better.

Model Predictive Control proves therefore to be a highly valuable tool in stabilizing humanoid walking robots which

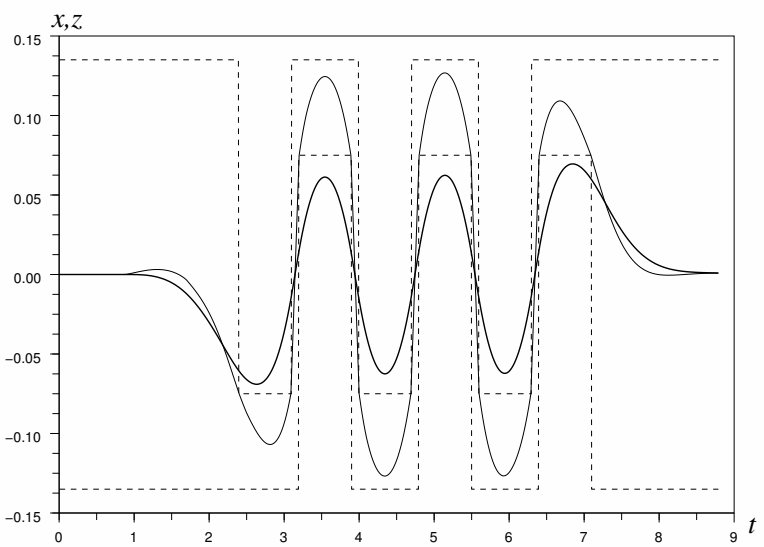

Fig. 7. Same results as in figure 1, but using a Quadratic Program with strict inequality constraints on the position of the CoP.

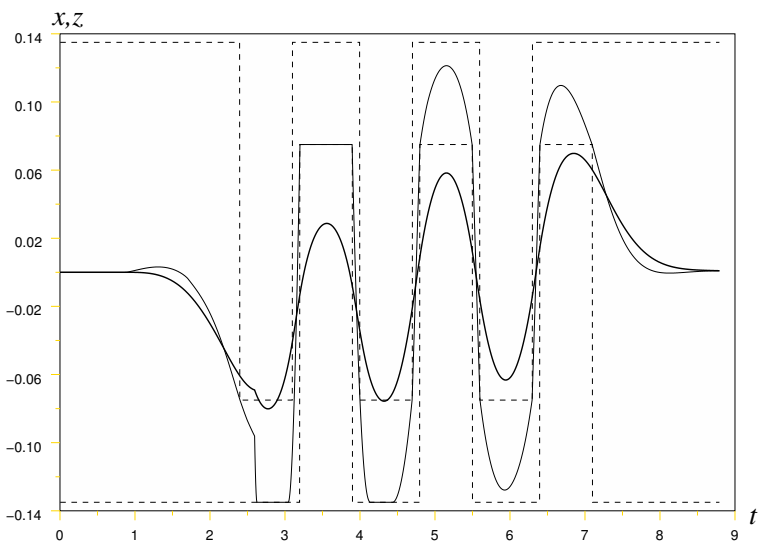

Fig. 8. Online recomputation of the trajectory of the Center of Mass of the robot with a Quadratic Program with strict inequality constraints on the position of the CoP, taking into account the perturbation at time $t=2.5 \mathrm{~s}$ generated by a mass corresponding to $33 \%$ of the total mass of the robot.

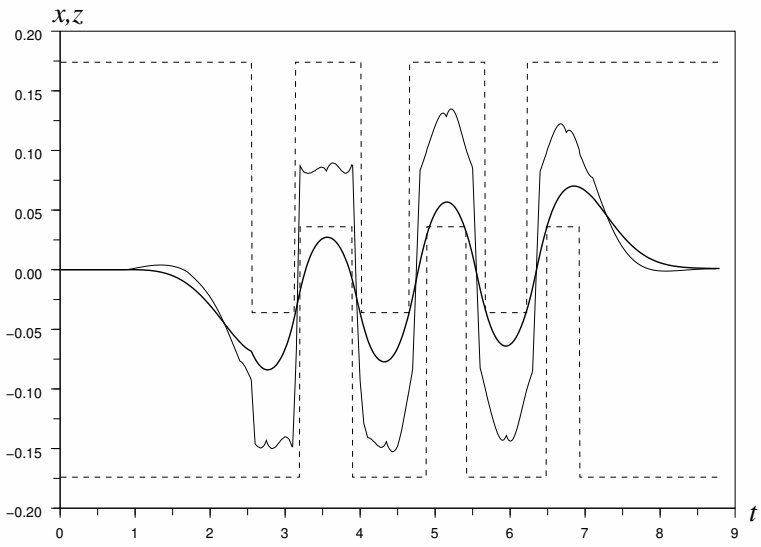

Fig. 9. Same results as in figure 8 , but with the position of the CoP (thin curve) evaluated this time with the whole dynamical model of the robot, and compared with the true limits on its position. 
are highly nonlinear dynamical systems submitted to severe limitations. More precisely, considering a minimization of the jerk of the trajectory of the Center of Mass of a humanoid robot over a finite horizon as in the ZMP Preview Control scheme allows generating stable walking motions which can be recomputed online, continuously taking into account the actual state of the robot. This allows compensating very strong perturbations, especially with the new scheme proposed in section $\mathrm{V}$ which explicitely takes care of the limits on the position of the Center of Pressure. This new scheme will be experimented soon on a real HRP-2 robot.

\section{ACKNOWLEDGMENTS}

Part of this study has been realized during a two months research visit in the AIST/CNRS Joint Robotics Lab in Tsukuba, Japan, thanks to the Post-Doctoral Fellowship Program from the Japanese Society for the Promotion of Science. It wouldn't have been possible without many fruitful discussions with all the people there and in the Humanoid Robotics Group.

\section{REFERENCES}

[1] P.-B. Wieber, "On the stability of walking systems," in Proceedings of the International Workshop on Humanoid and Human Friendly Robotics, 2002.

[2] K. Hirai, M. Hirose, Y. Haikawa, and T. Takaneka, "The development of Honda humanoid robot," in Proceedings of the IEEE International Conference on Robotics \& Automation, 1998.

[3] Q. Huang, K. Kaneko, K. Yokoi, S. Kajita, T. Kotoku, N. Koyachi, H. Arai, N. Imamura, K. Komoriya, and K. Tanie, "Balance control of a biped robot combining off-line pattern with real-time modification," in Proceedings of the IEEE International Conference on Robotics \& Automation, 2000.

[4] J. Park and H. Cho, "An on-line trajectory modifier for the base link of biped robots to enhance locomotion stability," in Proceedings of the IEEE International Conference on Robotics \& Automation, 2000.
[5] J. Yamaguchi, A. Takanashi, and I. Kato, "Development of a biped walking robot compensating for three-axis moment by trunk motion," in Proceedings of the IEEE/RSJ International Conference on Intelligent Robots \& Systems, 1993.

[6] P.-B. Wieber and C. Chevallereau, "Online adaptation of reference trajectories for the control of walking systems," To appear in Robotics and Autonomous Systems.

[7] D. Mayne and H. Michalska, "Receding horizon control of nonlinear systems," IEEE Transactions on Automatic Control, vol. 35, no. 7, pp. 814-824, 1990.

[8] D. Mayne, J. Rawlings, C. Rao, and P. Scokaert, "Constrained model predictive control: stability and optimality," Automatica, vol. 26, no. 6, pp. 789-814, 2000.

[9] D. Mayne, "Control of constrained dynamic systems," European Journal of Control, vol. 7, pp. 87-99, 2001.

[10] C. Azevedo, P. Poignet, and B. Espiau, "Moving horizon control for biped robots without reference trajectory," in IEEE International Conference on Robotics and Automation, Washington, USA, May 2002, pp. 2762-2767.

[11] S. Kajita, F. Kanehiro, K. Kaneko, K. Fujiwara, K. Harada, K. Yokoi, and H. Hirukawa, "Biped walking pattern generation by using preview control of zero-moment point," in Proceedings of the IEEE International Conference on Robotics \& Automation, 2003.

[12] K. Harada, S. Kajita, K. Kaneko, and H. Hirukawa, "An analytical method on real-time gait planning for a humanoid robot," in International Conference on Humanoid Robotics, 2004.

[13] S. Kagami, T. Kitagawa, K. Nishiwaki, T. Sugihara, M. Inaba, and H. Inoue, "A fast dynamically equilibrated walking trajectory generation method of humanoid robot," Autonomous Robots, 2002.

[14] O. Stasse, A. Davison, R. Zellouati, and K. Yokoi, "Real-time 3d slam for humanoid robot considering pattern generator information," in Proceedings of the IEEE/RSJ International Conference on Intelligent Robots \& Systems, 2006.

[15] K. Nishiwaki and S. Kagami, "High frequency walking pattern generation based on preview control of zmp," in Proceedings of the IEEE International Conference on Robotics \& Automation, 2006.

[16] P.-B. Wieber, "Constrained dynamics and parametrized control in biped walking," in Proceedings of the International Symposium on Mathematical Theory of Networks and Systems, 2000. 